\title{
Association of built environment with physical activity and among Chinese women
}

\section{CURRENT STATUS: POSTED}

Research Square

Yuliang Sun

Shaanxi Normal University

ORCiD: https://orcid.org/0000-0001-9071-7379

Chunzhen He

Shaanxi Normal University

Zhiwei Cheng

Shaanxi Normal University

Jianyu Hou

Shaanxi Normal University

Fangjun Sun

Shaanxi Normal University

Xinxin Zhang

Shaanxi Normal University

Wenfei Zhu

vwzhu@snnu.edu.cnCorresponding Author

ORCiD: https://orcid.org/0000-0002-6660-6350

\section{DOI:}

10.21203/rs.2.14906/v2

\section{SUBJECT AREAS}

Maternal \& Fetal Medicine

\section{KEYWORDS}

Built Environments, Physical Activity, Health 


\section{Abstract}

The authors have withdrawn this preprint from Research Square 